\title{
Comparison of Radiation Therapy Alone and Concurrent Chemoradiation Therapy for in Operable Cervical Cancer.
}

Md. Zillur Rahman Bhuiyan ${ }^{1}$, Atiar Rahman², Sarwar Alam, Towfiqul Hasan Firoz ${ }^{4}$, Sheikh Saiful Islam Shaheen ${ }^{5}$, Joly Bishwas $^{6}$

\footnotetext{
${ }^{1}$ Assistant Professor, Department of Oncology, ${ }^{2}$ Assistant Professor, Department of Transfusion Medicine, ${ }^{3}$ Associate Professor, Department of Oncology, BSMMU ${ }^{4}$ Registrar, Department of Radiation Oncology, National Institute of Cancer Research \& Hospital, ${ }^{5}$ Medical Officer, Department of Transfusion Medicine, ${ }^{6}$ Professor, Department of Transfusion Medicine, BSMMU.
}

\begin{abstract}
:
Objectives: Concurrent Chemoradiation therapy (CCRT) is the standard treatment for inoperable cervical cancer. The purpose of this study was to compare the outcomes of CCRT with cisplatin and radiotherapy (RT) for inoperable cervical cancer. Methods: From January 2012 to June 2012, 60 female patients with FIGO stage IIB to IVA were enrolled in this randomized phase III trial to compare radiotherapy (RT) alone with chemo radiotherapy (RT 5 Fraction in a week and Cinplatin $40 \mathrm{mg}$ weekly). External beam radiation therapy was delivered using a telecobalt- 60 unit. This was followed by 21 Gy of intracavitary brachytherapy. Results: In total, 30 patients were recruited: RT alone (30) and CCRT (30). The age of patients ranged from 26-65 years. There was statistically significant (p value $<0.037$ ) complete response $(70.00 \%$ CCRT group vs $43.30 \%$ RT group) in CCRT group. Conclusion: CCRT was seen to have better efficacy for patients with inoperable cervical cancer than RT alone.
\end{abstract}

Key words: Inoperable cervical cancer, chemoradiation therapy, radiotherapy.

[BSMMU J $2014 ; 7(2): 84-90]$

\section{Introduction:}

The cervix is the important part of uterus. Its adjacent areas are the anterior, posterior and lateral vaginal fornices. Anteriorly the cervix is related to the base of the bladder and posteriorly the rectum and the pouch of Douglas. Laterally it is related to the ureters. The junction, between the primary colummar epithelium of the endocervix and squamous epithelium of the ectocervix is the site, where cervical carcinoma develops. Over $80 \%$ of tumors of the cervix are squamous cell carcinoma. Approximately $15 \%$ are classified as adenocarcinoma and other rare histological varieties include-adenosquamous carcinoma, adenoid cystic carcinoma, small cell carcinoma, undifferentiated carcinoma, sarcomas, malignant lymphomas (Primary and metastatic) and others ${ }^{1}$.

Worldwide, carcinoma of the uterine cervix or cervical carcinoma is the second most common cancer in women,

Address for Correspondence: Dr. Md. Zillur Rahman Bhuiyan Assistant Prof. (Oncology) \& Deputy Registrar (Additional charge) Registrar office, Room \# 223, Block- B, BSMMU. accounting for $15 \%$ of all malignancies. The incidence and mortality rates caries among the countries, the highest rates being recorded in the developing countries. The global yearly incidence of cervical cancer for 2002 was 493,200 ; the annual death rate was 273,500 . It is estimated that in the year 2005 , there were about 520,000 cervical cancer cases in the world, of which 443,000 were in the developing countries. Over $80 \%$ of the cervical cancer cases presents at a fairly advanced stage with five years survival rates less than $40 \%$. It is the most common cancer is the most frequent cause of cancer death in women. Although the mean age of diagnosis is 52 years, carcinoma of the urerine cervix can be found in women between the ages of 17 and 90 years ${ }^{2}$.

In Bangladesh, about 25000 women are diagnosed as carcinoma of uterine cervix every year. Mortality is very high; nearly half of them die annually. Frequency of carcinoma of the uterine cervix from hospital based registry is $22-29 \%$. International federation of Gynecologic \& Obstetrique (FIGO) clinical sage of disease at presentation is the most important predictor of long-term survival. 
Recurrencess more than five years after treatment are extremely rare 3 .

After establishing the diagnosis of invasive cervical cancer histologically, the disease is staged clinically. Patients with cervical carcinoma should be treated with close collaboration between the gynecologic oncologist and the radiation oncologist, and an integrated team approach should be vigorously pursued. Invasive cervical cancer may be treated by surgery, radiotherapy, or a combination of both, with or without chemotherapy. Surgical treatment (radical hysterectomy) is effective management for early stages of cervical cancer. Cervical carcinoma is one of the malignancies that have been effectively treated with radiotherapy. Radiotherapy involves using ionizing radiation to kill cancer cells and can be used to treat early as well as late stages of cervical cancer. Concurrent chemo-radiation should be considered in preference to radiation alone in the treatment of early stage carcinoma uterine cervix with poor prognostic factors after surgery and in the locally advanced cervical carcinoma. Chemotherapy is not used as a primary line of treatment for cervical cancer ${ }^{4}$.

Molecular and epidemiologic evidence clearly indicate that certain type of human papilloma virus (HPV), which is sexually transmitted, are the principal causes of invasive cervical cancer and cervical intraepithelial neoplasia (CIN). More than $100 \mathrm{HPV}-16$ and HPH-18 are types most commonly linked with cancer, present in $70 \%$ of cervical cancers and high-grade CINs. Two vaccines to prevent cervical cancer were approved by the FDA and became available in 2006 and 2009 respectively ${ }^{4}$.

Patients with cervical carcinoma should be treated in close collaboration with the gynecologic oncologist and the radiation oncologist, and an integrated team approach should be vigorously pursued. Invasive cervical cancer may be treated by surgery, radiotherapy, or a combination of both, with or without chemotherapy ${ }^{5}$.

Surgical treatment (radical hysterectomy) is effective management for early stages of cervical cancer. Cervical carcinoma is one of the malignancies that have been effectively treated with radiotherapy. Radiotherapy involves using ionizing radiation to kill cancer cells and can be used to treat early as well as late stages of cervical cancer.

Concurrent chemo-radiation should be considered in preference to radiation alone in the treatment of early stage carcinoma uterine cervix with poor prognostic factors after surgery and in the locally advanced cervical carcinoma. Chemotherapy is not used as a primary line of treatment for cervical cancer.

An updated RTOG trial for advanced cervical cancer stage (stage IIB-IVA disease) compared pelciv EBRT plus concurrent 5-FU and cisplatin with pelvic and paraaortic EBRT in both arms, these therapies were followed by intracavitary irradiation. The addition of chemotherapy to irradiation improved 5-years survival from $55 \%$ to $79 \%$ and disease-free survival from $46 \%$ to $74 \%$ for stage IB/IIA diseases by reducing the rates of both local recurrence and distant metastases. For stage III/IVA disease, chemo radiotherapy improved 5-year survival from $45 \%$ to $59 \%$ and disease-free survival from $37 \%$ to $54 \%{ }^{4}$.

The combination of concurrent weekly cisplatin and irradiation significantly reduced the relapse rate and improved survival by $50 \%$. The 3 -year survival tare was significantly improved from $74 \%$ to $83 \%$ with the use of chemotherapy; this improvement was primarily due to a reduced risk of local recurrence (21\% vs $9 \%$ ) The use of weekly cisplatin for 6 cycles or 5-FU and cisplatin every 3 weeks for 2 cycles concurrently with radiotherapy is the standard treatment approach for bulky stage IB2 cervical cancer ${ }^{4}$.

\section{Treatment:}

Injury to the gastro-intestinal tract usually appears with the first 2 years after radiation therapy, whereas complications of the urinary tract are more often seen 3 to 4 years after treatment ${ }^{6}$.

Rectal complications include bleening, stricture, ulceration, and fistula. Urinary tract complications include haematuria, fistula, and urethral stricture ${ }^{1}$.

Small bowel obstruction is an infrequent complications of standard radiotherapy for patients without special risk factors. Risk factions for this are trans-peritoneal lymph nose dissection. Pelvic inflammatory disease, thin body 
habitus, and large daily fraction sizes.

Most patients treated with radical radiotherapy have some agglutination and telangiectasia of the apical vagina. More significant vaginal shortening can occer, particularly in elderly, postmenopausal women and those with extensive tumors treated with a high dose of irradiation1.

Chemotherapy: Chemotherapy has traditionally been used for the palliative management of advanced or recurrent disease that can no longer be managed by surgery or radiation therapy ${ }^{4}$. Chemotherapy is now being more extensively used in bulky and advanced cervical cancer, primarily, because of less-than-satisfactory results of initial therapy with other modalities; chemotherapy may eventually play and important role in the management of several groups of patients, including those with advanced tumors, those with pelvic or para-aortic nodal metastases carrying a low potential for cure with current local treatment modalities, and those with recurrent disease, after surgery and radiation therapy.

Studies that have explored combinations of chemotherapy and radiation therapy for patients with locally advanced cervical cancers have generally used one of two strategies.

Primary goal of studies that combine radiation therapy (both external beam therapy and brachytherapy) with concurrent chemotherapy is to use chemotherapeutic agents to sensitize tumour cells to the effects of radiotherapy ${ }^{1}$.

Fifty-two drugs have been studied in sufficient numbers of patients with carcinoma of the cervix to asses their activity. Nineteen of these have yielded response rates (partial and complete) of least $15 \%$ and may be of therapeutic value.

Several of the platinum compounds have been evaluated in greater detail. Cisplatin has been studied in a variety of doses and schedules. These studies have demonstrated that cisplatin remains the platinum compound of choice for patients with cervical carcinomas.

Cisplatin: Cisplatin is unusual among modern pharmaceuticals in that it is based on a metallic element whereas most drugs are purely organic ${ }^{7}$.
Chemistry: Platinum may exist in six oxidation states-Pt (I to VI) Among these Pt (II) molecule with cisconfiguration has the potential anticancer activity. Discovery of cisplatin has revolutionized the treatment of many solid tumours.

\section{Methods:}

This phase III randomized clinical trial was conducted for a period of six months from January 2012 to June 2012 in 60 patients, with histological proven advanced inoperable cervical cancinoma enrolled from Bangabandh Sheikh Mujib Medical University, National Institute of Cancer Research \& Hospital and Dhaka Medical College \& Hospital, Dhaka, Bangladesh. Patients aged equal to or less than 70 years with stages IIB-IVA having normal hematological, renal and hepatic functions, Eastern Cooperative Oncology Group performance status up to scale 2, no prior chemotherapy, radiotherapy or surgery were eligible for this study. For exact clinical staging, all patients were subjected to a through physical examination and pelvic examination by radiation oncologists and gynecologic oncologist, complete blood count (CBC), lever function test, urine analysis, and imaging studies including chest X-ray, Ultrasonogram of abdomen, pelvic magnetic resonance imaging or positron-emission tomography-computed tomography scanning. Cystoscopy and sigmodoscopy were performed only in patients clinically suspicious of bladder and bowel incasion. After the complete work-up the patients were randomized and statified (by age and stage) into two groups: Radiotherapy (RT) alone and the Chemoradiation (CCRT) group, using a computer generated random numbers table.

Informed written consent was taken from every patient before starting the trial.

RT consisted of pelvic external-beam radiation therapy (EBRT) and brachytherapy. Pelvic EBRT was given to all patients with the four-field box tecnique at a daily dose of 1.8 grays (GY), 5 days a week of total 50.4 Gy in 28 fractions for $5 \frac{1}{2}$ weeks. This was delivered using a covalt- 60 unit with $80 \mathrm{~cm}$ cource to surface distance by anteroposterior/posteroanterior or four field box technique to the whole pelvis (typical field size is $16 \mathrm{~cm} \mathrm{x}$ $14 \mathrm{~cm})$. The upper border of the pelvic field was at the 
at the L4-L5 junction; the lower border was at lowest part of the obturator foramen, which was modified according to the vaginal extent of the disease. Lateral borders were kept $1.5 \mathrm{~cm}$ beyond the widest pelvic brim. When the four field box technique was used, the anterior or posterior borders of the lateral portals were kept at the anterior part of the pubic symphysis and the S2-S3 junction, respectively. External beam RT was followed by Intracavitary brachytherapy. It was given at high dose rate (HDR) 21 Gy in 3 fractions over 5-8 days at one weekly inerval to point $\mathrm{A}$ which was marked as $2 \mathrm{~cm}$ above and $2 \mathrm{~cm}$ lateral to the cervical os. Chemotherapy was given weekly with RT, that is, five cycles during EBRT. Cisplatin was given in a dose of $40 \mathrm{mg} / \mathrm{m} 2$ during external beam therapy in patients in the CRT group by slow i.v. infusion over 2 hours with appropriate hydration. Chemotherapy was administered within an hour prior to delivering the fraction of RT.

The response to concurrent chemotherapy and radiotherapy was assessed after completion of radiotherapy using physical examination, pap's smear, MRI, and/or PET-CT by a radiation oncologist and a gynecologic oncologist. Complete response was determined by a complete diaappearance of all measurable lesions for at least 1 month. Parrial response was a more than $50 \%$ reduction in lesion diameter with no demonstrable disease progression elsewhere. The revised National Cancer Institute (NCI) common toxicity criteria (CTC) version 2.0 was used to score all acute radiation ( $<90$ days) and chemotherapy toxicities associated with these protocol.

Case-control differences were evaluated using Student's t-test for continuous variables and $\mathrm{x} 2$ tests for categorical variables. All analyses were performed using SPSS 18 software. A p value of less than 0.05 was considered significant.

\section{Result:}

Within the study period, sixty consecutive patients met the eligibility criteria and were enrolled. Of them, thirty patients were to the radiotherapy (RT) group and thirty to the chemoradiation (CCRT) group. The age of patients ranged from 26-65 years with peak age incidence found in age group of 46-55 years. Regarding parity, it has been observed that majority of the patients of both groups were multiparous. $20(66.70 \%)$ women had given birth to 5-6 children in RT group and 19 (63.30\%) women in CCRT group. A good number of patients belonged to stage IIB (33.33\% in CRT group vs $30 \%$ in RT group). There was statistically significant $(\mathrm{p}$ value $<0.037$ ) complete response (70.00\% CRT group vs $43.30 \% \mathrm{RT}$ group) in CRT group. The types and frequencies of adverse effects are shown in Table 3. There were no treatment-related deaths. The major acute toxicities was more in CRT group than in RT group. The grade I nausea vomiting, grade I leucopenia and grade II anaemia were more observed in CCRT group than in RT group respectively ( 80.00 vs $53.30 ; 60.00$ vs $20.00 ; 66.70$ vs 40.00$)$. But there were no instances of serious late complications requiring laparotomy in CCRT group. No remarkable alopecia was observed, nor did cisplatin-induced neurotoxicity or ototoxicity occur. Irreversible adverse effects, including persistent electrolyte imbalance and bone marrow failure, were not noted in the both groups.

\section{Table-I}

Distribution of patients according to response pattern in both groups

\begin{tabular}{llll}
\hline $\begin{array}{l}\text { Response } \\
\text { after treatment }\end{array}$ & RT group & CRT group & \\
\hline Complete Response & $13(43.30)$ & $21(70.00)$ & 0.037 \\
Partial Response & $17(56.70)$ & $09(30.00)$ & \\
Total $\quad 30(100)$ & $30(100)$ & & \\
& & &
\end{tabular}


Table-II

Distribution of patients on the basis of staging and response

\begin{tabular}{ccccccc}
\hline & \multicolumn{3}{c}{ RT group } & \multicolumn{3}{c}{ CRT group } \\
\hline & Total $(\mathrm{n})$ & CR $\mathrm{n}(\%)$ & PR $\mathrm{n}(\%)$ & Total $(\mathrm{n})$ & CR $\mathrm{n}(\%)$ & PR $\mathrm{n}(\%)$ \\
IIB & 18 & $8(44.44)$ & $10(55.55)$ & 20 & $15(75.00)$ & $5(25.00)$ \\
IIIA & 6 & $4(66.66)$ & $2(33.33)$ & 5 & $4(80.00)$ & $1(20.00)$ \\
IIIB & 5 & $1(20.00)$ & $4(80.00)$ & 4 & $2(50.00)$ & $2(50.00)$ \\
IVA & 1 & $0(0)$ & $1(100)$ & 1 & $0(0)$ & $1(100)$ \\
\hline
\end{tabular}

$\mathrm{CR}=$ Complete response, $\mathrm{PR}=$ Partial Response

Table-III

Distribution of patients on treatment related toxicities according to common toxicity criteria (CTC) version 2.0

\begin{tabular}{lcccc}
\hline Toxicities & \multicolumn{2}{c}{ RT Group } & \multicolumn{2}{c}{ CRT Group } \\
& Number & percentage & Number & percentage \\
\hline Nausea/Vomiting & & & & \\
Grade 0 & 12 & 40.00 & 04 & 13.30 \\
Grade I & 16 & 53.30 & 24 & 80.00 \\
Grade II & 02 & 6.70 & 02 & 6.70 \\
Cutaneous & & & & \\
Reaction & 02 & 6.70 & 00 & 00 \\
Grade 0 & 07 & 23.30 & 05 & 16.70 \\
Grade I & 21 & 70.00 & 25 & 83.30 \\
Grade II & & & & \\
Hematological & & & & \\
& & 60.00 & 10 & 33.30 \\
Anaemia & 18 & 40.00 & 20 & 66.70 \\
Grade 0 & 12 & & & \\
Grade I & & & \\
\hline
\end{tabular}

\section{Discussion:}

Despite improvement in radiation equipment and tecniques, the radiation therapy alone to the pelvis fails to control locally advanced cervical carcinoma. Because of the presence of dose limiting organ, recto-sigmoid part of GIT (gatro-intestinal tract) and urinary bladder in proximity to the cervix, it is important to restrict radiation dose to this critical organs to $50 \mathrm{~Gy}$, which is the maximal tolerance dose (Total Dose 50/5) for it. Exceeding this maximal tolerance limit, probability of major morbidity and sometimes mortality increases. Bearing this fact in mind that tumour dose by external beam was decided to be $50 \mathrm{~Gy}$, Inj. Cisplatin was added with the idea that may enhance tumour cell killing of radiation by its radio sensitizing effects.

Chemoradiationtherapy has been a standard treatment in women with locally advanced cervical cancer since five large randomized trials reported it to be associated with improved progression-free survival and overall survival 12-16. In this study, the complete response rate to concurrent chemoradiation was $70 \%$. The response rate was about to similar with the results to Tseng et al. (90\%) or Kersh et al. (88\%).

GIT toxicity, Cutaneous toxicity and Anaemia were the principle adverse effects in our trail. The frequency of grade I in GIT and cutaneous toxicity and grade II in anaemia were more in CRT group. Even though acute toxicities were more in cisplatin given group, they were manageable. There was no patient death and drop out in this trial. Negi et al and Whitney et al found the similar findings as our study.

It must be stated before progressing any further that despite the author's utmost effort this Study was conducted over a limited period of time on a small number 
of patients.

The objectives of this study were to observe the immediate response of radiotherapy alone and concurrent chemo-radiation in locally advanced (inoperable) carcinoina of uterine cervix and to compare the effects of two regimens in tumour radiation reactions and other toxicities.

Because of the presence of dose limiting organ, rectosigmoid part of GIT (gastro-intestinal tract) behind the uterus, it is important to restrict radiation dose to this organ to $50 \mathrm{~Gy}$, which is the maxinial tolerance dose (TD 50/5) for it. Exceeding this maximal tolerance limit, probabillty of major morbidity and sometimes mortality, increases. Bearing thes fact in mind that tumour dose by external beam was decided to be $50 \mathrm{~Gy}$, Inj. Cisplatin was added with the idea that it may enhance turnour cell killing of radiation by its radio sensitizing effects.

Two arms were formed, arm-A and arm-B. 20 patients were enrolled in each arm. All patients in both arms received external beam radiation with 50 Gy in 25 daily fractions over five weeks. Followed by two insertions of brachytherapy each 500 Gy. Patients in arm-B received Inj. cisplatin in addition, as $25 \mathrm{mg} / \mathrm{m} 2$ IV infusion before radiotherapy on the first day of each treatment week.

In this study the age of the patients are ranged between 26 to 65 years. About $40 \%$ of the patients in arm-A belonged to $36-45$ years age group and $45 \%$ in arm-B belonged to 46-55 years age.

In this study most patients presented with the complaints of per vaginal (P/V) watery/white discharge, per vaginal bleeding, pelvic pain and anorexia. Out of 20 cases in arm-A P/V bleeding was present in 17 (85\%), P/V d1scharge (waery/white) in $20(100 \%)$, pelvic (lower abdominal) pain in $14(70 \%)$, anorexia in $14(70 \%)$ and urinary problem (dysurea /frequency of micturition) in 6 (30\%) cases. Out of 20 cases in arm-B P/V bleeding, was present in $16(80 \%)$, P/V discharge (watery/white) in 20 $(100 \%)$, pelvic pain in $16(80 \%)$, anorexia in $15(75 \%)$ and urinary problem in $13(65 \%)$ cases, These findings in clinical features had concordance with the findings.

It was observed that out of 20 cases in each arm the overall response (completeg partial response) in both groups were more or less equal. But complete response was observed in $13(65 \%)$ out of 20 cases in study group (arm-B), on the other the control group (arm-A) complete response was observed in $9(45 \%)$ of 20 . This difference in complete response in both groups was statistically significant $(\mathrm{p}<0.005)$. Partial response was observed in $7(35 \%)$ out of 20 cases in Arm B, and 11(55\%) out of 20 cases in arm-A.

Studies abroad have also demonstrated increased cytotoxicity when cisplatin is combined with radiation therapy8. One GOG (Gynaecologic Oncologic Group) trial with weekly Cisplatin combined with radiation in IIB-IIIB cervical cancer showed 5 year survival rate9. Study by Zak et al with cisplatin and irradiation, used concomitantly on mice showed substantial enhancement of cell killing and co-workers reported results in 118 patients with invasive cervical carcinoma treated with 50 to $60 \mathrm{~Gy}$ to the whole pelvis. With doses of less than 50 Gy pelvic tumour control was not sicynificant but disease control and survival rates were significantly enhanced with higher doses 10. Other studies showed that local failure rates were significantly lower in patients who received radiation combined with cisplatin, which suggests that chemotherapy was acting as a radiation sensitizer ${ }^{11}$.

Based on the trial that compared cisplatin based chemo radiation with radiation alone, the National Cancer Institute (USA) released a clinical announcement to the incorporation of, concurrent chemo radiation for patients who require radiation therapy for management of cervical cancer.

About toxicity no severe untoward reaction was noted in most patients. Nausea and vomiting were more common in arm-B than arm-A. And these were well managed with conservative treatment in both arms. Skin reaction was observed more in arm-B than arm- $\mathrm{A}$, but there was no significant difference. Study showed that toxicity of 
single agent cisplatin combined with radiation was less.

After careful consideration of the above facts, it is evident that the patients who had pelvic irradiation and concurrent chemotherapy with cisplatin had better treatment response. In comparison to patients who received radiation alone. This statement is with the result showed in abroad were the statement is "Cisplatin containing chemotherapy when given concurrently with radiation have $30 \%-50 \%$ reduction in relative risk of relapse or death $^{11}$.

Due to short period of the study, overall long term survival rate could not be evaluated, these patients were kept for long term follow up for future study and to determine survival rate with a view to fulfill the secondary objective of the study.

From the result of the present findings as well as the findings obtained by a number of investigations, it is conceivable that concurrent chemo-radiation is more effective than radio-therapy alone in advanced cervical cancer. However, further studies are recommended to determine the efficacy of concurrent chemo-radiotherapy.

\section{Conclusion:}

In conclusion, in patients with locally advanced cervical cancer, there is a potential improvement with weekly cisplatin along with concurrent RT having acceptable toxicity. Further multicenter randomized trials with a number of newer chemotherapeutic agents are necessary to evaluate the overall oncologic outcomes and qualityof-life.

\section{Reference:}

1. Eifel JP, Berek JS, Jhigpen JT: Cancer of the cervix, cagina \& vulva PP. 1433-1455. In Cancer: Principles and Practice of Oncology. Ed 5 Devita VT, Hellmans, Rqsenberg SA. LippincotRaven, Philadelphia 1997.

2. Rubin Philip MD, Jacqueline P.Williams $\mathrm{PhD}$ editors. Clinical Oncology, A Multidisciplinary Approach for Physicians and Students, 8th edition, W.B. Saunders Company, 2001; pp 463-478.

3. FIGO Committee on Gynecologic Oncology. Revised FIGO staging flor carcinoma of the vulva, cervix, and endometrium. International Journal of Gynecology and Obstetrics 105(2009)103-104.

4. Pazdur R, Wagman LD, Camhauscn KA, Hoskin WJ (eds) 2010, Cancer Management: A Multidisciplinary Approach, 13th edition, 2010; UBM Medica LLC, Newyork; pp 473-507.

5. Bomford C.K, Kunkler IH, Sheriff SB, Miller H. (editors). Walter and Miller's Textbook of Radiotherapy, Radiation Physics, Therapy and Oncology, 6th edition. Edinburg: Churchil Livingstone 2003 ; p. 465-477.

6. Perez CA, 1998: Chap 62 In: Perez CA, Brady LW (eds.): Principles and Practice of Radiation Oncology, Ed. 3, LippincotRaven 1997; PP. 1733-1819.

7. Dwyer PJ, Johnson SW, Hamilton TC (eds.): Cisplatin and its analogues, pp, 418-428. In Cancer: Principles and Practice of oncology, ed 5 Devita VT, Helimans, Rqsenberg SA. LippincotRaven, Philadelphia 1997.

8. Britten RA, Evans AJ, Allalunis- Turner MJ, et al: Effect of cisplatin on the Clinically relevant radio sensitivity of human cervical carcinoma cell lines. Int. J Radiat Oncol Biol Phys 1996; 34: 367-374.

9. Rose PG, Bundy BN, Walkins EB, et al: Concurrent cisplatin based chemotherapy and radiotherapy for locally advanced cervical cancer, N Eng J Med 1999, 340:1144-1153.

10. Castro JR Issa P, Flecher GH: Carcinoma of the cervix treated by external irradiation alone. Radiology 1970;95: 163-166.

11. Peters WA III, Liu PY, Barrett R, et al: Cisplatin, 5-flurouracil plus radiation therapy are superior to radiation therapy as superior to radiation therapy as adjunctive therapy in high-risk, early-stage carcinoma of the cervix after radical hysterectomy and pelvic lymphadenectomy. Proc Soc Gynecol Oncol 199:28:1069. 